\title{
CONSIDERACIONES PARA LA INSTALACIÓN \\ DE UNA PLANTA PRODUCTORA \\ DE UN COMPLEMENTO NUTRICIONAL \\ PARA NIÑOS
}

LoREnA Godefroy, MARgarita BRICEÑO*

Universidad de Lima

Recibido: 12 de junio del 2018 / Aprobado: 5 de septiembre del 2018

doi: 10.26439/ing.ind2019.n037.4548

RESUMEN: En el Perú existe el problema de mala nutrición infantil. La presente investigación busca demostrar la viabilidad social, técnica y económica de un producto dirigido al mercado infantil con alto valor nutricional, que utilice parte de sus ganancias para financiar un programa para combatir la desnutrición infantil crónica al interior del país. Se usaron herramientas de la ingeniería industrial para determinar la demanda del mercado, la formulación técnica del producto, el tamaño y diseño de la planta de producción, el análisis económico y financiero y el impacto social.

Palabras clave: nutrición / impacto social / cereales andinos

CONSIDERATIONS TO SET UP A MANUFACTURING

PLANT FOR PRODUCING A NUTRITIONAL SUPPLEMENT FOR CHILDREN

ABSTRACT: Child malnutrition is a current problem in Peru. This research aims to demonstrate the social, technical and economic feasibility of manufacturing a nutritional product for children, and using part of the profits to support a social program to reduce chronic child malnutrition in the country's provinces. To this end, industrial engineering tools were used to calculate the market demand, the technical specifications of the product, the size and design of the manufacturing plant, the economic and financial analysis, and the social impact.

Keywords: nutrition / social impact / Andean cereals

*lorena.godefroy@gmail.com, margaritabricenom@gmail.com 


\section{INTRODUCCIÓN}

En el Perú coexisten dos problemas de alimentación infantil: por un lado, desnutrición crónica en las poblaciones más pobres y, por el otro, falta de opciones saludables en las ciudades más desarrolladas, lo cual causa obesidad infantil. Esta también afecta a los más pobres, llegando a un 11,9\% de incidencia en el quintil V (INS, 2012).

La desnutrición incrementa la mortalidad, morbilidad y tiene resultado adverso sobre el proceso de aprendizaje. La principal causa es una ingesta insuficiente de nutrientes, tales como carbohidratos, proteínas, minerales, vitaminas y minerales. En el Perú, la desnutrición crónica afecta al $19,5 \%$ de los niños menores a 5 años; los más afectados son aquellos en el quintil inferior de riqueza, en áreas rurales de la sierra y de la selva y con madres sin educación. Los departamentos más afectados son Huancavelica, Huánuco y Apurímac (INEI, 2012, p. 300).

La tasa de sobrepeso y obesidad en los niños de 0 a 5 años ha venido en aumento, con 5,1 \% en 1992, 5,5\% en 1996 y 6 \% en el 2007 (Ministerio de Salud, 2012). La obesidad infantil se ha asociado con la aparición de alteraciones metabólicas como diabetes, hipertensión, dislipidemia a edades más tempranas, hígado graso, apnea de sueño, asma y estrés mecánico. Las principales causas de la obesidad se asocian a un componente genético y a un estilo de vida donde priman el sedentarismo, patrones de alimentación con regímenes hipercalóricos y pobre ingesta de nutrientes esenciales.

El proyecto plantea ofrecer un producto que mejore ambos problemas alimenticios, enmarcados en el término "niños con mala nutrición". Se propone elaborar un complemento nutricional natural instantáneo para niños hecho a base de cereales andinos. El proyecto creará una empresa social que genere ingresos mejorando la calidad de la alimentación infantil en los niveles socioeconómicos (NSE) A, B y C del Perú para, con esas utilidades, brindar la nutrición requerida a poblaciones con pocos recursos (Huancavelica) y resolver una necesidad de poblaciones menos favorecidas.

¿Qué significa que sea "una empresa social"? Una empresa social es aquella que nace para resolver un problema social y cuyo fin social es tan importante como el económico. Se diferencia de una organización sin fines de lucro porque busca generar ingresos que sean sostenibles en el tiempo utilizando las herramientas de negocio.

\section{DESCRIPCIÓN DEL PRODUCTO}

El producto que se va a elaborar es un complemento nutricional instantáneo para niños hecho a base de cereales andinos. Como producto básico tenemos el complemento nutricional instantáneo homogéneo hecho con $15 \%$ harina de quinua, $15 \%$ harina de kiwicha, $30 \%$ de leche en polvo y $40 \%$ de cocoa. El producto real incluye presentación en stand-up pouches de polietileno de $200 \mathrm{~g}$ con cierre hermético y rotulado conforme 
a la ley. El producto aumentado incluye garantía de calidad, atención al cliente, página en internet y el aporte social que cada compra provee al proyecto de lucha contra la desnutrición crónica en Huancavelica. A continuación se describe el aporte nutricional:

\subsection{Quinua}

Es un cereal andino que "cuenta con un contenido de proteína superior a otros cereales, teniendo un rango entre 14 y $16 \%$, y proporciona una cantidad ideal de aminoácidos esenciales para el consumo humano" (Mestanza, Riegel, Silva y Vásquez, 2015). El 37 \% de las proteínas que posee la quinua son aminoácidos: ácido glutámico (producción de energía para el cerebro y aprendizaje), ácido aspártico, isoleucina, lisina (crecimiento muscular), fenilalanina, tirosina y valina, siendo los cuatro últimos, aminoácidos esenciales. Además contiene grasas mono y poliinsaturadas, es rica en fibra, libre de gluten y contiene 1,5 veces más calcio que el trigo.

Tabla 1

Análisis nutricional de la quinua blanca (base $100 \mathrm{~g}$ )

\begin{tabular}{lclc}
\hline Contenido & Valor & Elemento & Valor \\
\hline Calorías & $376 \mathrm{Cal}$ & Calcio & $120 \mathrm{mg}$ \\
Agua & $10,1 \mathrm{~g}$ & Fósforo & $165 \mathrm{mg}$ \\
Proteínas & $11,5 \mathrm{~g}$ & Hierro & $0 \mathrm{mg}$ \\
Grasas & $8,2 \mathrm{~g}$ & Retinol & $0 \mathrm{mcg}$ \\
Carbohidratos & $66,7 \mathrm{~g}$ & Vit.B1 & $0,12 \mathrm{mcg}$ \\
Fibra & $5,1 \mathrm{~g}$ & Vit.B2 & $0,14 \mathrm{mcg}$ \\
Ceniza & $3,5 \mathrm{~g}$ & Vit.B5 & $1,35 \mathrm{mcg}$ \\
\hline
\end{tabular}

Fuente: Soluciones Prácticas, s. f.

\subsection{Kiwicha}

La kiwicha tiene un valor energético comparativamente mayor que, por ejemplo, el maíz (14,5 de proteínas contra $10 \%$, respectivamente). El balance de aminoácidos de la kiwicha es cercano al requerido para la nutrición humana, siendo el limitante la leucina. Contiene ácido graso oleico y linolénico. 
Tabla 2

Análisis nutricional de la kiwicha (base $100 \mathrm{~g}$, tostada)

\begin{tabular}{lccc}
\hline Elemento & Valor & Elemento & Valor \\
\hline Calorías & $428 \mathrm{cal}$ & Calcio & $283 \mathrm{mg}$ \\
Agua & $0,7 \mathrm{~g}$ & Fósforo & $502 \mathrm{mg}$ \\
Proteínas & $14,5 \mathrm{~g}$ & Hierro & $8,1 \mathrm{mg}$ \\
Grasas & $7,8 \mathrm{~g}$ & Ácido ascórbico & $0,5 \mathrm{mcg}$ \\
Carbohidratos & $74,3 \mathrm{~g}$ & Vitamina B1 & $0,01 \mathrm{mcg}$ \\
Fibra & $3,0 \mathrm{~g}$ & Vitamina B2 & $0,01 \mathrm{mcg}$ \\
Ceniza & $2,7 \mathrm{~g}$ & Vitamina B5 & $1,30 \mathrm{mcg}$ \\
\hline
\end{tabular}

Fuente: Soluciones Prácticas, 2013

\subsection{Leche}

Es el insumo que más calorías aporta a la mezcla, así como proteínas y lisina, aminoácido importante para el crecimiento, desarrollo óseo y absorción del calcio.

\subsection{Cocoa}

Dado que el producto está dirigido a niños, debe contar con un sabor agradable. El cacao en polvo puro tiene propiedades beneficiosas, como mejorar la función cerebral, bajar el colesterol "malo" y estabilizar la presión arterial. Para obtener el cacao en polvo se parte del cacao en polvo crudo y se refina a altas temperaturas, de modo que se reducen ligeramente sus propiedades nutricionales.

\subsection{Complemento nutricional}

La empresa Certificaciones Peruanas (CERPER, 2016) analizó una muestra del producto y se obtuvieron los resultados de la tabla 3.

Tabla 3

Especificaciones técnicas del producto (base: $1 \mathrm{~kg}$ del complemento nutricional)

\begin{tabular}{lll}
\hline Ensayos & Prueba utilizada & Información nutricional por $100 \mathrm{~g}$ \\
\hline Humedad $(\mathrm{g} / 100 \mathrm{~g})$ & $\begin{array}{l}\text { Método gravimétrico } \\
\text { NTP 209.264. } 2013\end{array}$ & $4,60 \mathrm{~g}$ \\
\multirow{2}{*}{ Proteína $(\mathrm{g} / 100 \mathrm{~g})(\mathrm{N} \times 6,25)$} & $\begin{array}{l}\text { Método Kjeldahl de proteína } \\
\text { NTP 209.262.2013 }\end{array}$ & $19,39 \mathrm{~g}$ \\
\hline
\end{tabular}

(continúa) 
(continuación)

\begin{tabular}{|c|c|c|}
\hline Grasa (g/100g) & $\begin{array}{l}\text { Método gravimétrico } \\
\text { NTP 209.263. } 2013\end{array}$ & $11,73 \mathrm{~g}$ \\
\hline Ceniza (g/100g) & $\begin{array}{l}\text { Método gravimétrico } \\
\text { NTP 209.265. } 2013\end{array}$ & $6,25 \mathrm{~g}$ \\
\hline $\begin{array}{l}\text { Hierro }(\mathrm{mg} / \mathrm{kg}) \\
\text { (LC: } 0,56 \mathrm{mg} / \mathrm{kg})\end{array}$ & $\begin{array}{l}\text { Método de espectrofotometría } \\
\text { de absorción atómica }\end{array}$ & $12,99 \mathrm{mg}$ \\
\hline $\begin{array}{l}\text { Zinc }(\mathrm{mg} / \mathrm{kg}) \\
\text { LC: } 1,26 \mathrm{mg} / \mathrm{kg}\end{array}$ & NOM-117-SSA1. 1994. & $4,05 \mathrm{mg}$ \\
\hline $\begin{array}{l}\text { Calcio }(\mathrm{mg} / \mathrm{kg}) \\
(\mathrm{LC}: 41,27 \mathrm{mg} / \mathrm{kg})\end{array}$ & & $772,82 \mathrm{mg}$ \\
\hline Carbohidratos $(\mathrm{g} / 100 \mathrm{~g})$ & Por cálculo & $58,03 \mathrm{~g}$ \\
\hline Calorías (Kcal/100g) & & $415,25 \mathrm{kcal}$ \\
\hline $\begin{array}{l}\text { Calorías provenientes de las } \\
\text { proteínas (Kcal/100g) }\end{array}$ & & $77,56 \mathrm{kcal}$ \\
\hline $\begin{array}{l}\text { Calorías provenientes de las } \\
\text { grasas (Kcal/100g) }\end{array}$ & & $105,57 \mathrm{kcal}$ \\
\hline $\begin{array}{l}\text { Calorías provenientes de los } \\
\text { carbohidratos (Kcal/100g) }\end{array}$ & & $232,12 \mathrm{kcal}$ \\
\hline
\end{tabular}

Nota: LC: Límite de cuantificación.

Fuente: Cerper, 2016

\section{ESTUDIO DE MERCADO}

Para determinar la demanda del producto en estudio se siguió la siguiente metodología.

\subsection{Definir y analizar el mercado objetivo}

El mercado objetivo son niños (de 2 a 12 años, excluyendo al periodo de lactancia) de los NSE A, B y $C$ del Perú. Se utilizan datos de NSE utilizando como fuentes a Ipsos, APEIM, INEl; estudios de mercado correspondientes a preferencias del consumidor, perfil del consumidor y del niño, características de los hogares, hábitos de compra y estadísticas poblacionales del Perú.

\subsection{Clasificar comercialmente al producto y obtener la demanda interna aparente}

Con la clasificación comercial se puede obtener la competencia y, con ello, las exportaciones, importaciones y producción nacional de una categoría. El producto se define como complemento nutricional natural, hecho con insumos nacionales ("cereales andinos"). 
Los principales competidores serían Kiwigen y Nesquik Cereales Andinos, los cuales se producen internamente. Cabe resaltar que no se encontraron importaciones al analizar la data. Con ello se obtiene la demanda interna aparente (DIA) del producto en estudio.

Tabla 4

Demanda interna aparente en $\mathrm{kg}$ por año

\begin{tabular}{lccccc}
\hline & 2011 & 2012 & 2013 & 2014 & 2015 \\
\hline Importaciones & 0 & 0 & 0 & 0 & 0 \\
Producción & 434412 & 463378 & 473101 & 497806 & 546308 \\
Exportaciones & 37556 & 42141 & 46313 & 59000 & 112644 \\
DIA & 396856 & 421237 & 426788 & 438806 & 433665 \\
\hline
\end{tabular}

Fuente: Sunat, 2016

\subsection{Encuesta exploratoria al mercado objetivo}

La encuesta es aleatoria, empleando un procedimiento probabilístico de muestreo estratificado, por lo que el resultado será representativo. La población objetivo son niños y su madre, padre o apoderado, de NSE A, B y C. Se aplica la encuesta en Lima Moderna porque, dado que el $98 \%$ de su población pertenece a los NSE A, B y C, el encuestado tiene altas probabilidades de pertenecer a los NSE objetivo. El tamaño de la muestra depende del nivel de confianza $(95 \%, Z=1,96)$, error $(0,05)$ y variabilidad positiva $(60 \%$ de aceptación en una encuesta previa):

$$
\eta=\frac{\rho \times q \times z^{2}}{E^{2}}=\frac{0,6 \times(1-0,6) \times 1,96^{2}}{0,05^{2}}=368,8
$$

Se deberían aplicar 369 encuestas pero, dado que la investigación no es un estudio de factibilidad sino de prefactibilidad, la cantidad de encuestas a aplicar es de alrededor de 150. En total, se aplicaron 178 encuestas, de las cuales 130 aceptaron el producto (82\%). A los encuestados que aceptaron el producto se les preguntó la intensidad de compra, en una escala del 1 al 10, teniendo como promedio un 7,4 de intensidad.

\subsection{Demanda específica del proyecto}

Finalmente, la DIA obtenida es segmentada de acuerdo al mercado objetivo y a los resultados de la encuesta. El mercado objetivo son niños entre 2 y 12 años de los NSE A, B y C. La proporción de dichos segmentos es se muestra en la tabla 5. 
Tabla 5

Porcentaje de población del Perú perteneciente a NSE A, B y C

\begin{tabular}{cc}
\hline Año & Porcentaje NSE A, B y C \\
\hline 2011 & $29,0 \%$ \\
2012 & $34,4 \%$ \\
2013 & $34,4 \%$ \\
2014 & $37,2 \%$ \\
2015 & $37,8 \%$ \\
\hline
\end{tabular}

Elaboración propia con datos de Ipsos, 2015

La segmentación demográfica de "niños entre 2 y 12 años" no se considerará debido a que la venta de los productos Kiwigen y Nesquik, datos con los que se halló la DIA, ya se encuentra segmentada a niños. En base a los resultados de la encuesta, el $82 \%$ sí compraría el producto con una intensidad media de 7,4. El resultado de aceptación e intensidad es de $61 \%$.

Tabla 6

Demanda específica del proyecto del 2011 al 2015

\begin{tabular}{cc}
\hline Año & Demanda específica $(\mathrm{kg})$ \\
\hline 2011 & 70204 \\
2012 & 88392 \\
2013 & 89557 \\
2014 & 99574 \\
2015 & 99994 \\
\hline
\end{tabular}

Elaboración propia

Se proyecta la demanda del 2016 al 2025 con ajuste lineal (figura 1, tabla 7).

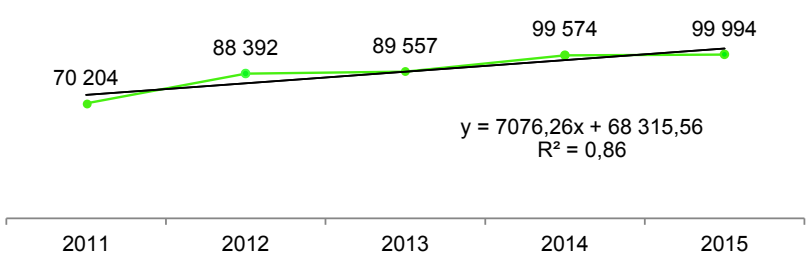

Figura 1. Demanda específica del proyecto del 2011 al 2015 (kg)

Elaboración propia 
Tabla 7

Demanda específica del proyecto del 2016 al 2025 ( $\mathrm{kg} / a$ ño)

\begin{tabular}{cc}
\hline Año & Demanda específica $(\mathrm{kg})$ \\
\hline 2016 & 110773 \\
2017 & 117849 \\
2018 & 124926 \\
2019 & 132002 \\
2020 & 139078 \\
2021 & 146154 \\
2022 & 153231 \\
2023 & 160307 \\
2024 & 167383 \\
2025 & 174459 \\
\hline
\end{tabular}

Elaboración propia

\section{TECNOLOGÍA DEL PROCESO}

\subsection{Almacenamiento de materia prima e insumos}

El proceso comienza con la recepción de insumos, se realiza un control de calidad. Los insumos serán recibidos en sacos y almacenados en el almacén de materias primas.

\subsection{Pesado inicial}

El proceso de producción será por lotes. Se realizará un pesado inicial del lote utilizando una balanza digital.

\subsection{Extruido}

La extrusión se realiza solo a los cereales quinua y kiwicha. Los granos pasan por sistema de cocción de alta temperatura y compresión, junto con cizallamiento durante periodos cortos. El proceso se desarrolla entre 150 y $160^{\circ} \mathrm{C}$ y a 1,2 atm de presión. Antes de ingresar a la extrusora el grano debe hidratarse hasta $15 \%$ de humedad durante 25 minutos. La duración de la extrusión es de entre 5 y 12 segundos, de acuerdo a la cantidad ingresada. La masa obtenida es cortada por una cuchilla rotatoria. El producto obtenido tiene entre 10 y $11 \%$ de humedad. 


\subsection{Molido y tamizado}

Los cereales extruidos ingresan al molino de martillos por la tolva superior. Luego ingresan a una cámara que cuenta con martillos de alto impacto que trituran los granos hasta que puedan pasar a través del tamiz encontrado en la parte inferior. Este tamiz tiene una malla ASTM E-11/05 (N.o 120) de $125 \mu \mathrm{m}$. A la salida del molido y tamizado se realizará un control de calidad para verificar la granulometría de la quinua y kiwicha antes de pasar al mezclado.

\subsection{Dosificado}

El dosificador volumétrico es accionado por un motor eléctrico que controla la velocidad del tornillo dosificador de salida, de manera que se puede programar el caudal de salida por lotes para llegar a la proporción final adecuada. En esta etapa se incluye la leche en polvo y la cocoa en las proporciones requeridas.

\subsection{Mezclado}

En la mezcladora horizontal un agitador genera el movimiento de las dos cintas helicoidales, una interior y una exterior; esta última posee un diámetro y alcance mayor. La mezcla homogénea de quinua, kiwicha, leche en polvo y cocoa terminada se descarga por una compuerta inferior.

\subsection{Envasado}

La máquina envasadora automática contiene una tolva donde se coloca el producto mezclado y, por otro lado, es alimentado por una película plana dispuesta en forma de bobina, la cual vendría a ser el empaque de polietileno. El avance de la lámina se efectúa mediante rodillos laterales de tracción accionados por motor y el resto de los movimientos es neumático. Una vez terminado el sellado se depositan las bolsas en una paila y son movidas a la zona de encajado. Se colocan 20 bolsas en una caja y, tras una inspección final de calidad, se procede a sellarlas. El llenado de los sacos de $10 \mathrm{~kg}$ destinados al Programa Nutri+ (ver sección 6), se realizará de manera manual con ayuda de una balanza. Tanto las cajas selladas como los sacos de papel en parihuelas son llevados al almacén de productos terminados. 


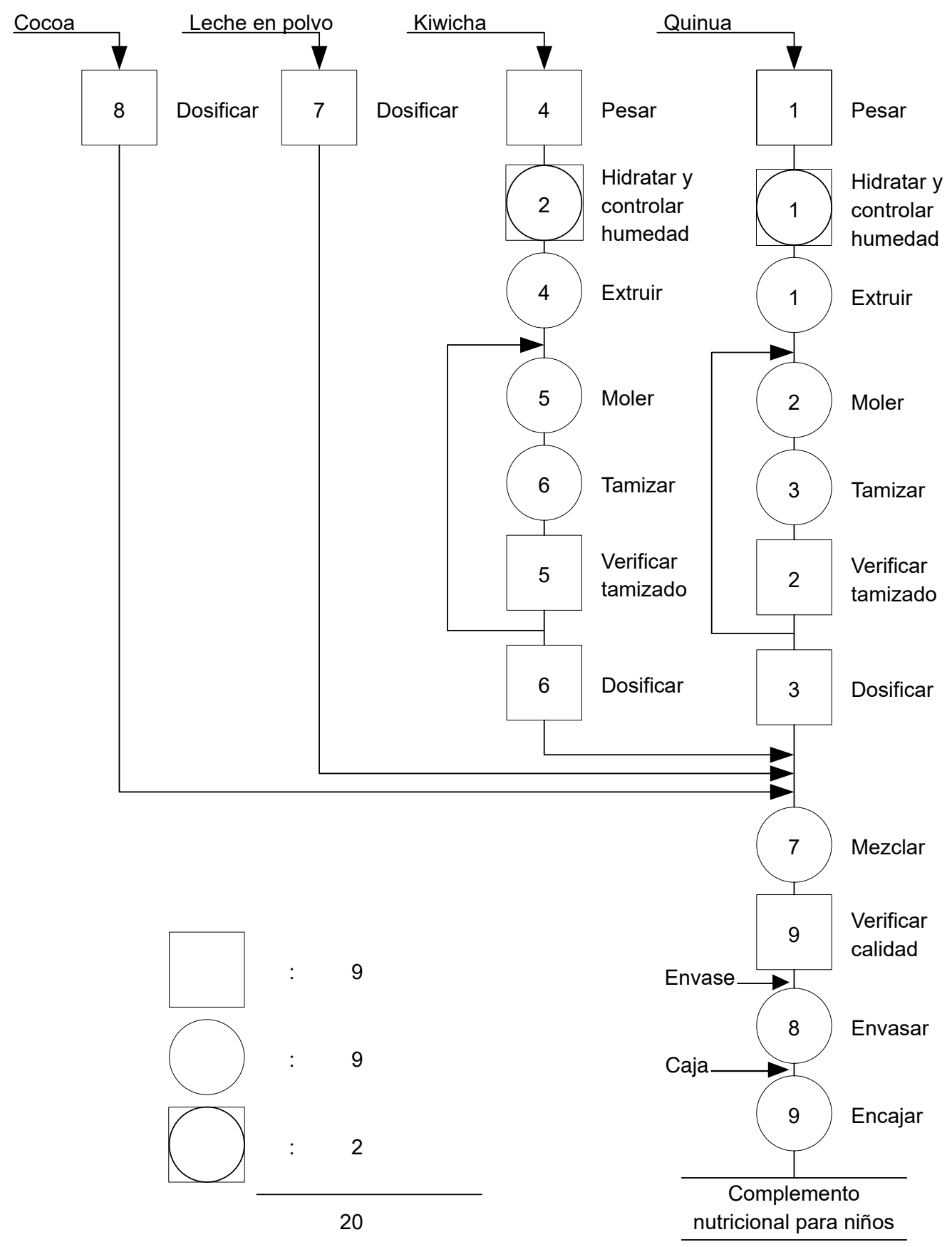

Figura 2. Diagrama de operaciones del proceso de elaboración del complemento nutricional Elaboración propia 


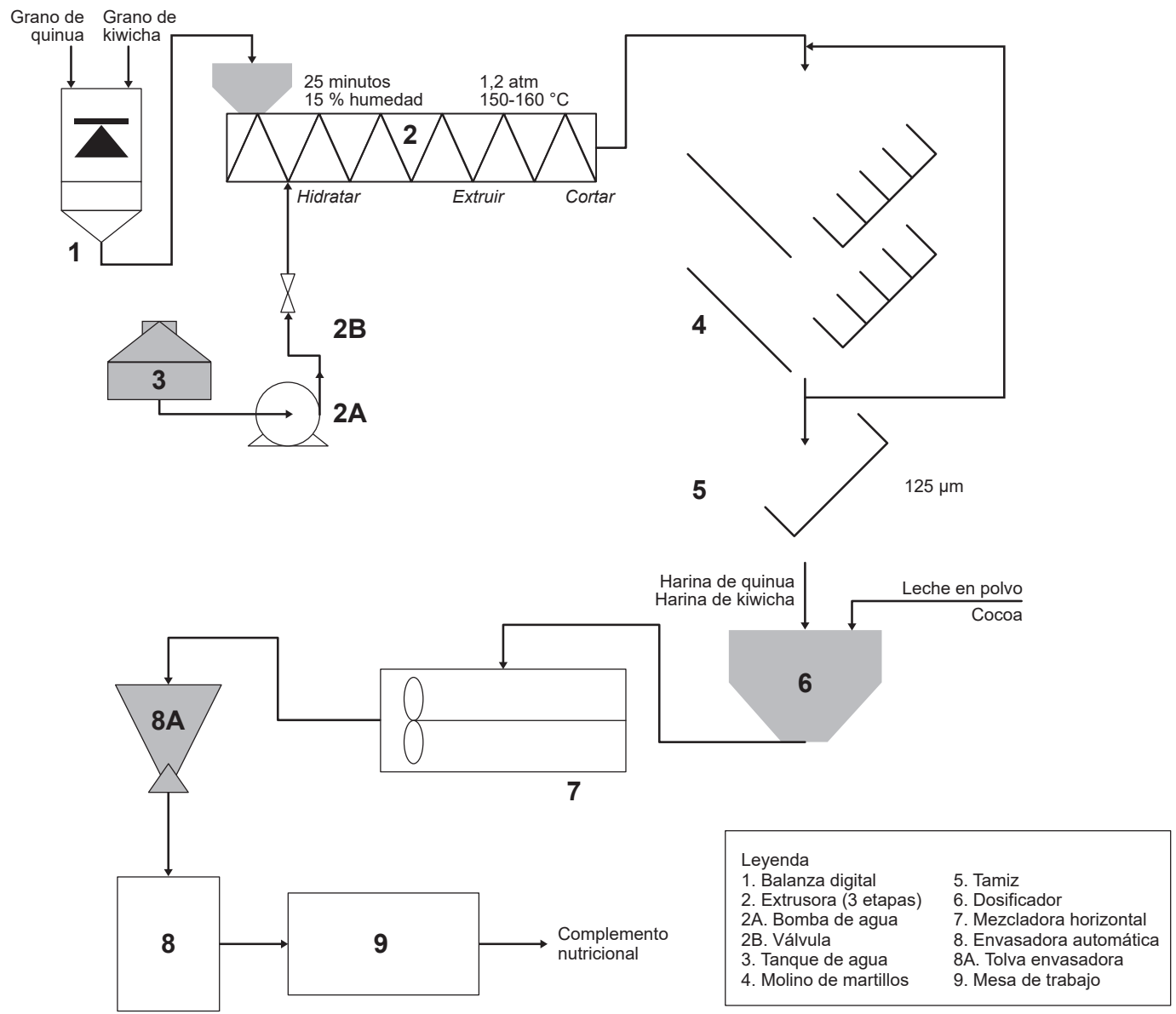

Figura 3. Diagrama de flujo del proceso de elaboración de complemento nutricional Elaboración propia 


\section{DISEÑO DE LA PLANTA DE PRODUCCIÓN}

La planta estará localizada en el distrito de Villa El Salvador, en Lima Metropolitana. La decisión se basó en comparar factores como distancia a los almacenes de los distribuidores, disponibilidad y costo del terreno, acceso a energía y agua, y facilidad de obtener permisos municipales.

Se diseña la planta teniendo como tamaño requerido la producción del último año del proyecto, en el cual la demanda es mayor: $174459 \mathrm{~kg}$ de producto. El programa de producción incluye inventario de seguridad y el producto destinado al programa social Nutri+ (ver sección 6).

Tabla 8

Programa de producción anual ( $\mathrm{kg})$

\begin{tabular}{lcccccccccc}
\hline & 2016 & 2017 & 2018 & 2019 & 2020 & 2021 & 2022 & 2023 & 2024 & 2025 \\
\hline $\begin{array}{l}\text { Inventario } \\
\text { inicial }\end{array}$ & 0 & 318 & 339 & 359 & 379 & 400 & 420 & 440 & 461 & 481 \\
$\begin{array}{l}\text { Demanda } \\
110773\end{array}$ & 117849 & 124926 & 132002 & 139078 & 146154 & 153231 & 160307 & 167383 & 174459 \\
Nutrit & & & 2356 & 2569 & 2800 & 3052 & 3326 & 3626 & 3952 & 4308 \\
Producción & 111092 & 117870 & 127302 & 134591 & 141898 & 149226 & 156577 & 163953 & 171356 & 178285 \\
$\begin{array}{l}\text { Inventario } \\
\text { seguridad }\end{array}$ & 318 & 339 & 359 & 379 & 400 & 420 & 440 & 461 & 481 & 502 \\
$\begin{array}{l}\text { Inventario } \\
\text { final }\end{array}$ & 318 & 339 & 359 & 379 & 400 & 420 & 440 & 461 & 481 & 0 \\
\hline
\end{tabular}

Elaboración propia

En base a la producción anual y a la capacidad de cada máquina, se determina el número de máquinas requerido, siempre en función de la producción del último año.

Con la cantidad de máquinas y equipos se determina el área mínima de producción mediante el método de Guerchet, el cual considera el área de la maquinaria, el espacio requerido para operarlas y el uso de elementos móviles. El área requerida solo para la zona de producción es de $53,40 \mathrm{~m}^{2}$.

Además de la zona de producción, la planta requiere de un almacén de insumos y de productos terminados, área de control de calidad, baño de planta, oficinas administrativas, un comedor, patio de maniobras, zona de desechos y finalmente una caseta de seguridad. Tomando en cuenta espacios para desplazamiento y disposición, la planta tendría un área de 28 por $21 \mathrm{~m}$ o $588 \mathrm{~m}^{2}$. 
Tabla 9

Número de máquinas requeridas

\begin{tabular}{lccccc}
\hline \multicolumn{1}{c}{ Máquina } & $\begin{array}{c}\text { Capacidad } \\
(\mathrm{kg} / \mathrm{hora})\end{array}$ & $\begin{array}{c}\text { Q procesado } \\
(\mathrm{kg})\end{array}$ & $\mathrm{U}$ & $\mathrm{E}$ & N. ${ }^{\circ}$ Máq. \\
\hline Balanza & 150 & 55043,3 & 0,95 & 0,83 & 1 \\
Extrusora de 3 etapas & 80 & 55875,8 & 0,95 & 0,83 & 1 \\
Molino de martillos & 65 & 55595,8 & 0,96 & 0,83 & 1 \\
Malla ASTM E-11/95 & 65 & 55039,8 & 0,96 & 0,83 & 1 \\
$\begin{array}{l}\text { Dosificador volumétrico } \\
\text { de tornillos }\end{array}$ & 271 & 182598,7 & 0,92 & 0,83 & 1 \\
$\begin{array}{l}\text { Mezcladora de cinta } \\
\text { horizontal }\end{array}$ & 300 & 182001,0 & 0,90 & 0,83 & 1 \\
Envasadora automática & 200 & 179271,0 & 0,97 & 0,83 & 1 \\
\hline
\end{tabular}

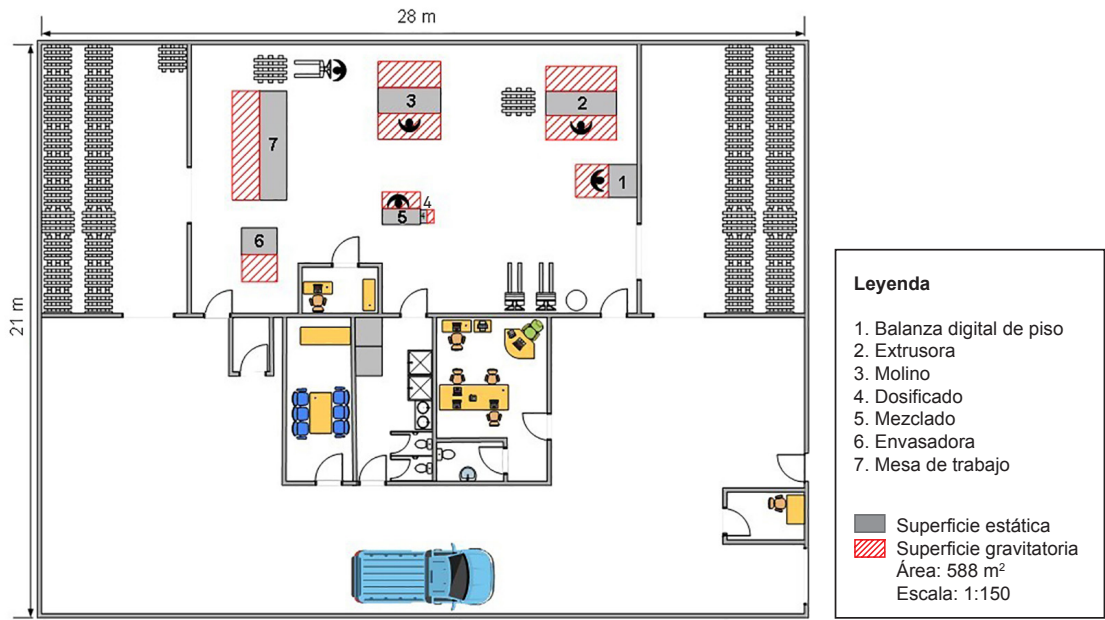

Figura 4. Plano de la planta

Elaboración propia

\section{IMPACTO SOCIAL}

Como se mencionó al principio, la empresa en estudio es una empresa social. El problema al que busca contribuir es combatir la desnutrición infantil en zonas menos favorecidas. Para cumplir con ello se implementó un plan integral en una zona que tenga un alto índice de desnutrición crónica infantil. En base a dicho factor y la distancia a Lima, se escogió como primera localidad la provincia de Huaytará, en Huancavelica.

Huancavelica fue seleccionada porque tiene el índice de desnutrición infantil más alto $(42,4 \%)$, seguida por Cajamarca $(35,6 \%)$ (INEI, 2009), y Huaytará porque la desnutrición infantil supera el 50 \%, muy por encima del promedio del departamento. 


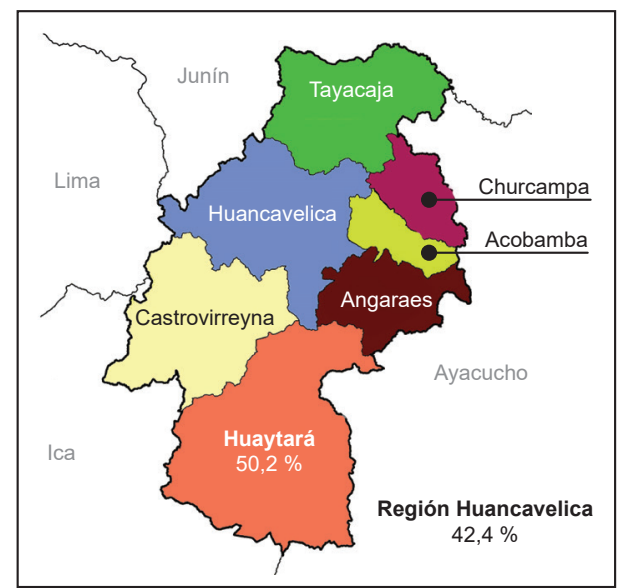

Figura 5. Mapa del índice de desnutrición crónica infantil de Huancavelica Fuente: INEI, 2009

\subsection{Entrega de complemento alimenticio y lavado de manos}

El distrito escogido es Santiago de Chocorvos, con un número potencial de niños para el programa de 1076. Se apunta a llegar al $25 \%$ del total, lo cual equivale a 269 en el 2018, e irá incrementando a razón de $0.9 \%$ al año, promedio del incremento anual de la población de Huancavelica (INEI, 2015).

La ejecución del programa incluye listar a los niños con su DNI o DNI de la madre, evaluación médica de los principales indicadores de desnutrición para el registro de estatura, peso, nivel de vitamina A, yodo y hierro. Estos indicadores de seguimiento serán medidos por un médico cada tres meses para obtener los impactos del programa.

En cada localidad se va seleccionar a una madre del programa: la promotora Nutri+. Ella será encargada de promocionar la asistencia para la entrega de los productos, revisión de la limpieza de manos y las capacitaciones de los padres. Además, será encargada del registro de asistencia, limpieza de manos, preparación, entrega y almacenamiento del producto. Este puesto será cambiado cada mes en coordinación con la municipalidad de Santiago de Chocorvos.

La entrega del desayuno será diaria en el centro, facilitada por la municipalidad. Los asistentes deben firmar la carta de asistencia, lavarse las manos de la forma en la que han sido capacitados. El desayuno consiste de una dosis del complemento nutricional $(24 \mathrm{~g}$ ), una fruta y un huevo. Los costos y gastos descritos serán cubiertos por las ganancias de la venta del producto en Lima Metropolitana. Se puede apreciar que, por los primeros dos años de vida del proyecto, no se realizará el Programa Nutri+ para así poder generar suficiente flujo de efectivo y en el tercer año poder implementarlo. Se presenta el detalle en la tabla 10. 


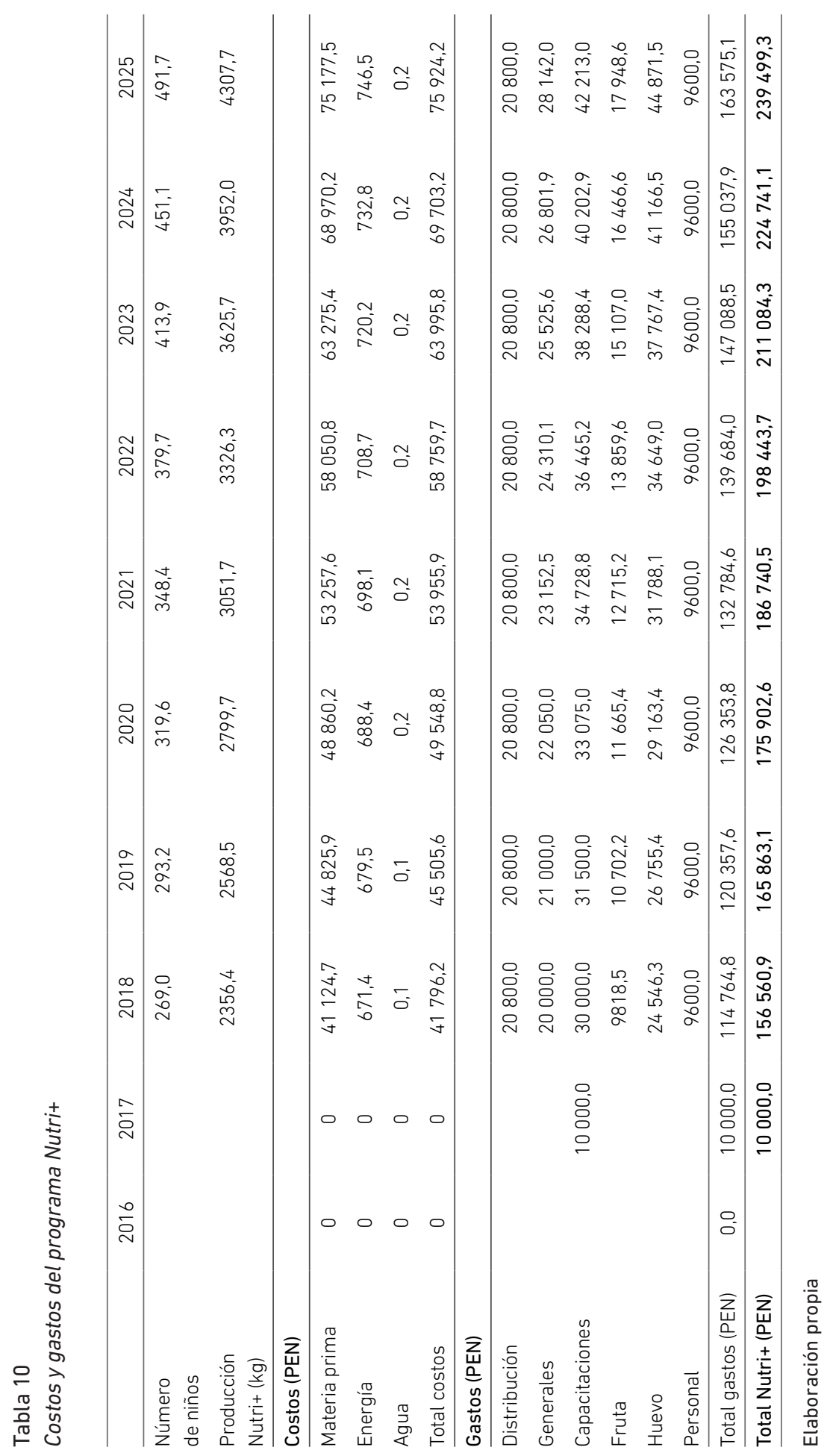




\subsection{Capacitación de nutrición para los padres}

Se programarán capacitaciones en los centros vecinales de los distritos vinculados o colegios donde se realizan las entregas del complemento, de forma trimestral. Además, todo el año estará disponible la entrega de material educativo por si las madres tienen consultas. Las capacitaciones están dirigidas a los padres de la zona y tendrán siempre la siguiente temática:

- La importancia de la lactancia materna exclusiva por seis meses.

- La importancia de una buena alimentación en el periodo de embarazo y lactancia.

- Educación sobre cocina saludable y balanceada para madres y niños.

- Higiene personal: lavado de manos y su importancia para tener una familia sana.

- Planificación familiar.

Para el desarrollo del material se utilizará el conocimiento de expertos y recursos del Estado como los generados por la Comisión de la Dieta Andina Nacional (platos económicos con ingredientes nacionales) y los manuales de limpieza de manos de Essalud.

\section{VIABILIDAD ECONÓMICA Y FINANCIERA}

Para analizar la viabilidad económica y financiera, primero se determina la inversión inicial (tabla 11).

Tabla 11

Inversión inicial total

\begin{tabular}{ll}
\hline Tipo de inversión & Costo (PEN) \\
\hline Activos tangibles & 172373 \\
Activos intangibles & 326121 \\
Capital de trabajo & 258749 \\
\hline Total & 756749 \\
\hline
\end{tabular}

Elaboración propia

Los activos tangibles incluyen maquinaria, equipos, muebles, equipo ofimático e imprevistos; los intangibles, estudios previos, puesta en marcha, gastos preoperativos e inversión en software. La inversión tiene una relación deuda-capital de 1,5, es decir $60 \%$ préstamo y $40 \%$ capital social. El préstamo es a cinco años de cuotas constantes y una TEA de 15,82 \% (Superintendencia de Banca, Seguros y AFP, 2016). 


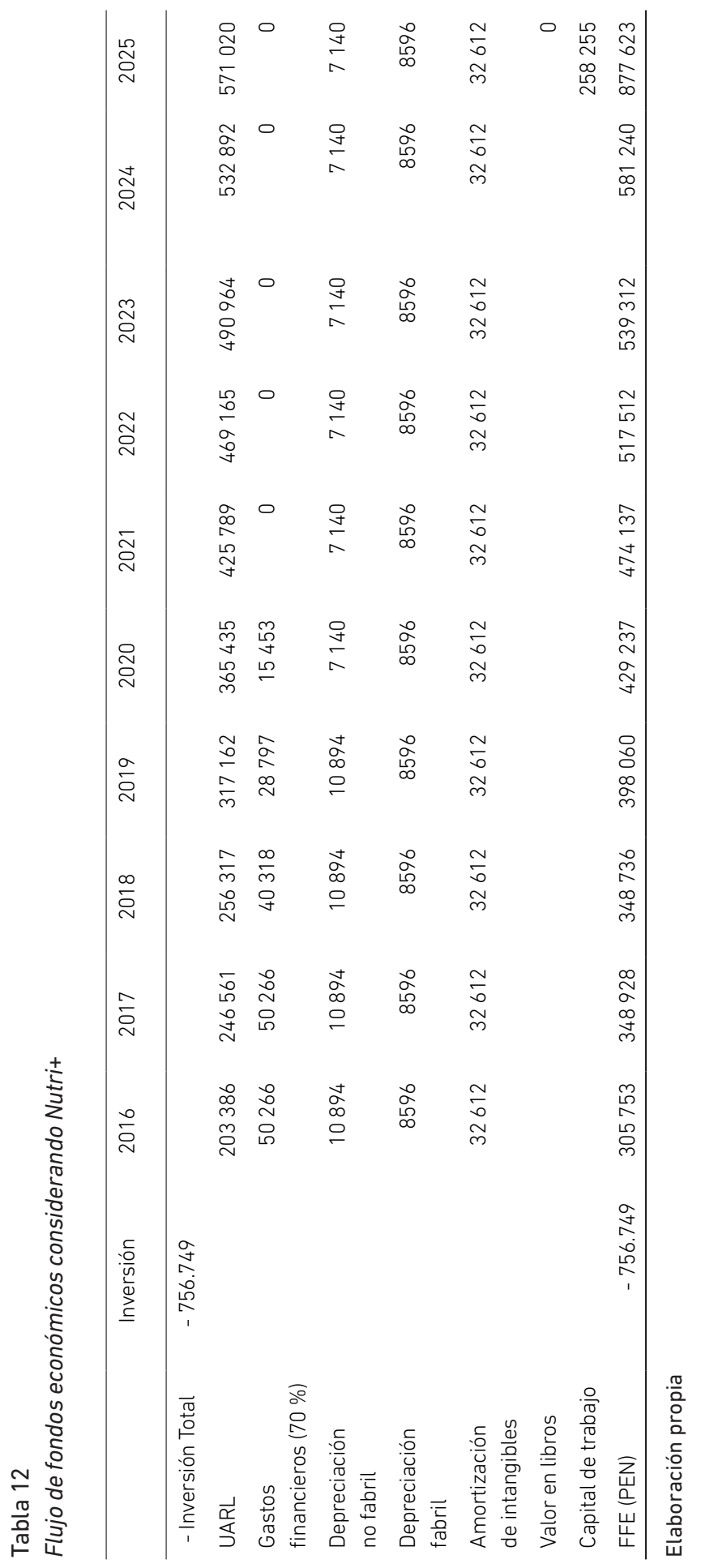




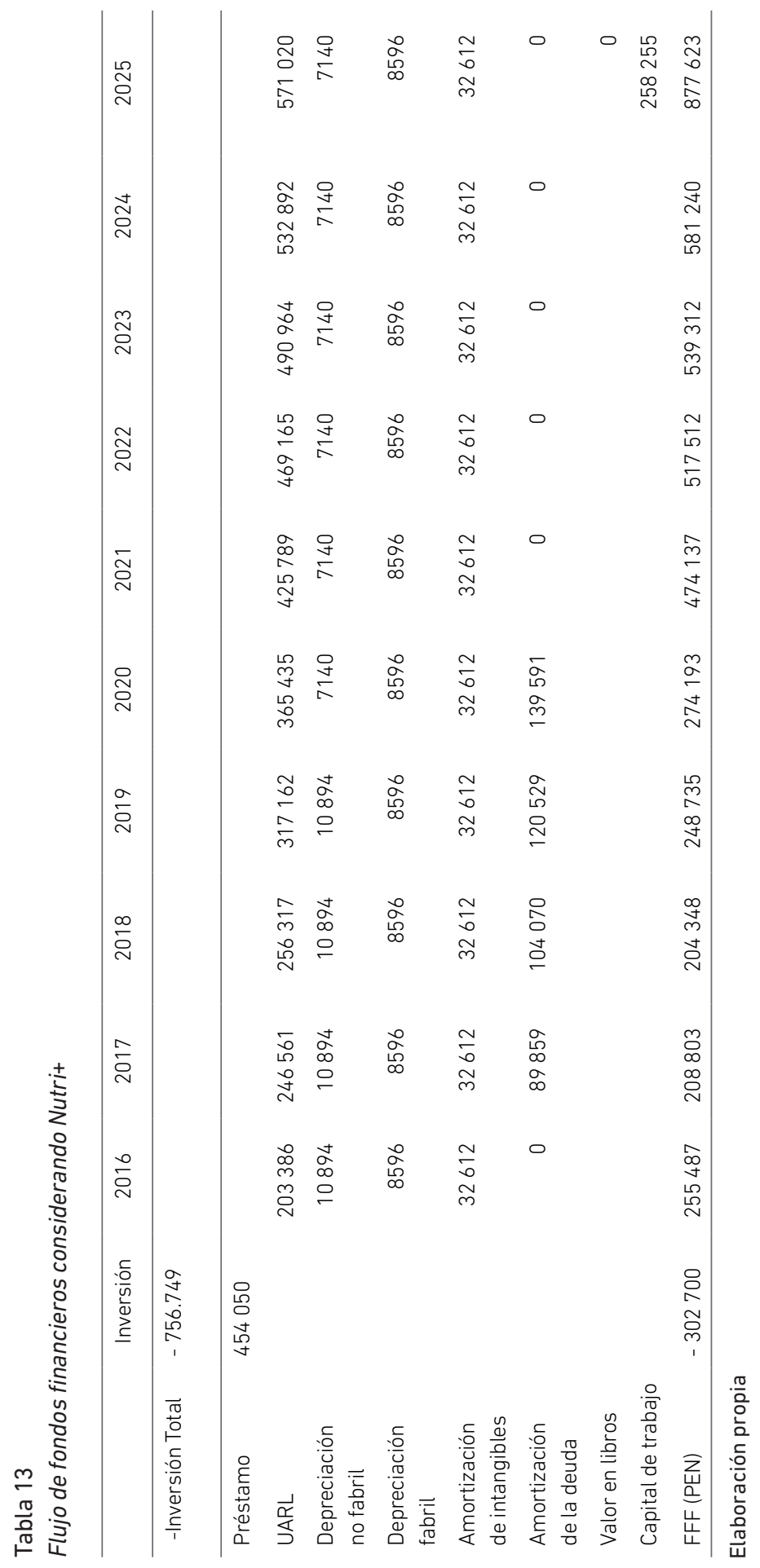


Los ingresos de la empresa están determinados por la venta del complemento nutricional, cuyo precio de venta al público es de PEN 10,50 por cada empaque de $200 \mathrm{~g}$, precio definido en base al estudio de mercado y de la competencia.

Por otro lado, dentro de los gastos se tiene el costo de ventas (materia prima, insumos, servicios de planta, mano de obra, mantenimiento, alquiler fabril), los gastos administrativos (legal, contable, tecnológico), de marketing, logísticos, depreciación y gastos financieros (producto del préstamo obtenido).

Para analizar la viabilidad económica y financiera se toma en cuenta el retorno esperado de los accionistas [COK] a través del método de Modelo de Valoración de Activos Financieros: $\mathrm{COK}=r_{f}+\beta_{\text {proy }} \times\left[r_{m}-r_{f}\right]$. El COK del proyecto es de $17,56 \%$.

En una empresa social, el resultado social y económico son igual de importantes. El fin social es importante puesto que es la razón de ser de la empresa y guía sus decisiones, pero la rentabilidad económica es la que determina si la empresa puede seguir funcionando, escalando y generando impacto. En las tablas 12, 13 y 14 se demuestra que una empresa con fin social puede ser rentable para los accionistas.

Tabla 14

Evaluación económica y financiera considerando Nutri+

\begin{tabular}{lcc}
\hline & Evaluación económica & Evaluación financiera \\
\hline VNA & PEN 1 930 917,10 & PEN 1 550 628,46 \\
VAN & PEN 1 174 167,77 & PEN 1 254 928,73 \\
TIR & $48 \%$ & $82 \%$ \\
B/C & 2,55 & 5,12 \\
P.R. & 4,14 & 2,56 \\
\hline
\end{tabular}

Elaboración propia

El programa Nutri+ es económica y financieramente rentable.

\section{CONCLUSIONES}

La instalación de una planta productora de complemento nutricional para niños es técnica y comercialmente viable porque existe un mercado interesado en el producto y la tecnología disponible para su realización.

El proyecto es económicamente viable porque los indicadores son positivos: la tasa interna de retorno es mayor al costo de oportunidad de los accionistas, el valor actual neto es mayor a cero, el beneficio costo es mayor a 1 y el periodo de recupero es inferior al tiempo de vida útil del proyecto. 
Los resultados económicos son positivos aun cuando el proyecto tiene un fin social, teniendo el potencial de impactar a cientos de niños en Huancavelica.

\section{REFERENCIAS}

Certificaciones Peruanas S. A. (CERPER) (2016). Informe de Ensayo N. ${ }^{\circ} 3-08780 / 16$. El Callao: Perú.

Instituto Nacional de Estadística e Informática (INEI) (2009). Encuesta demográfica y de salud familiar 2009. Visión nacional y departamental. Recuperado de https:// www.inei.gob.pe/

Instituto Nacional de Estadística e Informática (INEI) (2012). Encuesta demográfica y de salud familiar (ENDES) 2012. Nacional y departamental. Recuperado de https:// www.inei.gob.pe/

Instituto Nacional de Estadística e Informática (INEI) (2015). Población 2000 al 2015. Recuperado de http://www.inei.gob.pe/web/poblacion/

Instituto Nacional de Estadística e Informática (INEI) (2016). Producción manufacturera: producción de la industria de productos alimenticios y bebidas 2007-2012. Recuperado de https://www.inei.gob.pe/

Instituto Nacional de Salud (2012). Boletín INS (Perú) 18(11-12), p. 205.

Ipsos Apoyo (2012a). Estadística poblacional 2012. Lima.

Ipsos Apoyo (2012b). Niveles socioeconómicos de Lima Metropolitana 2012. Informe Gerencial de Marketing. Lima.

Ipsos Apoyo (2013). Niveles socioeconómicos de Lima Metropolitana 2013. Informe Gerencial de Marketing. Lima.

Ipsos Apoyo (2014). Niveles socioeconómicos de Lima Metropolitana 2014. Informe Gerencial de Marketing. Lima.

Ipsos Apoyo (2015). Niveles socioeconómicos de Lima Metropolitana 2015. Informe Gerencial de Marketing. Lima.

Mestanza, C., Riegel, R., Silva, H. y Vásquez, S. (2015). Characterization of the acetohydroxyacid synthase multigene family in the tetraploide plant Chenopodium quinoa. Recuperado de http://www.sciencedirect.com/science/article/pii/ S0717345815000962

Ministerio de Salud (2012). Un gordo problema: Sobrepeso y obesidad en el Perú. Recuperado de http://bvs.minsa.gob.pe/local/MINSA/1830.pdf 
Soluciones Prácticas (2013). Kiwicha. Valor nutricional. Recuperado de http://quinua.pe/ kiwicha-valor-nutricional/

Soluciones Prácticas (s. f.). Quinua. Valor nutricional. Recuperado de http://quinua.pe/ quinua-valor-nutricional/

Superintendencia Nacional de Aduanas y Administración Tributaria (Sunat) (2016). Operatividad aduanera. Recuperado de http://www.sunat.gob.pe/operatividadaduanera/index.html

Superintendencia de Banca, Seguros y AFP (2016). Tasa de interés promedio. Recuperado de http://www.sbs.gob.pe/

\section{BIBLIOGRAFÍA}

Bazile. D. et al. (2014). Estado del arte de la quinua en el mundo en 2013. Santiago de Chile: FAO.

Briceño, M. y Godefroy, L. (2017). Estudio de pre factibilidad para la instalación de una planta productora de un complemento nutricional para niños (tesis para optar el título de Ingeniero Industrial). Universidad de Lima.

Fondo Nacional de Desarrollo de la Educación Peruana (2016). Mapa de la desnutrición infantil en el Perú. Recuperado de http://www.fondep.gob.pe/?p=1638

Instituto Interamericano de Cooperación para la Agricultura (IICA) (2015). El mercado y la producción de quinua en el Perú. Recuperado de http://www.iica.int/es/content/ el-mercado-y-la-producci\%C3\%B3n-de-quinua-en-el-per\%C3\%BA

Instituto Nacional de Tecnología Industrial (2016). La extrusión como tecnología flexible de procesamiento de alimentos. Recuperado de http://www4.inti.gov.ar/gd/ jornadas2000/Pdf/cempam-064.pdf

Ocaña, J. (2012). Estudio de la aceptación de una bebida instantánea en base de semillas de quinua (Chenopodium quínoa) y amaranto (Amaranthus cruentus) para niños de edad escolar. (Trabajo de Investigación en Ingeniería de Alimentos). Universidad Técnica de Ambato, Ecuador). Recuperado de http://repositorio.uta.edu.ec/ jspui/handle/123456789/2030 
